\section{Ventilator-associated pneumonia: when is a pathogen not a pathogen?}

\author{
Michael J Cox
}

The context of respiratory microbiology is changing. 16S rRNA gene sequencing is a workhorse method in the field of environmental microbiology, established for over 30 years. ${ }^{1}$ Rather than requiring growth of organisms on agar plates it targets the DNA of all the microorganisms present, revealing bacteria irrespective of their particular growth requirements. Together with metagenomics, the untargeted shotgun sequencing of DNA extracted from a sample, these techniques have been applied to the respiratory tract. These approaches have revealed a characteristic respiratory community of microorganisms, a respiratory microbiota that varies in different diseases. $^{2-6}$ The most prevalent organisms in the healthy respiratory tract are Streptococcus spp., Veillonella spp. and Prevotella spp.; the latter two genera being anaerobic bacteria, intolerant of oxygen and not commonly isolated by classic microbiology approaches.

At times studies that use these techniques might seem to be just describing long lists of bacterial names, but sampling over time reveals dynamic interactions between organisms and with their environment and the word 'community' is therefore used deliberately. Not all of these changes in the presence and abundance of these organisms are immediately explicable; they might relate to any one of an enormous number of variables not measured by clinical studies. Forced expiratory volume has very little direct impact on a microorganism in the respiratory tract, whereas the presence of a particular microbial nutrient will.

Molecular methods, such as $16 \mathrm{~S}$ rRNA gene sequencing, have a different resolution to clinical culture. They are capable of sensitively detecting bacteria without the narrow selection of the limited range of culture conditions commonly used. Conversely, they are not always capable of providing identification beyond the level of genus. Where microbiota analyses really excel is in providing a full microbial context for disease, without a priori assumption of which organisms should be

Correspondence to Dr Michael J Cox, Section of Genomic Medicine, National Heart and Lung Institute, Imperial College London, London SW3 6LY, UK; michael.cox1@imperial.ac.uk targeted. They also remove some of the uncertainties of isolation of bacteria; for example, when patients are being treated with antibiotics, culture might prove negative despite other evidence for infection. As we're using the method to examine a community, they are at heart ecological analyses and reveal the impact of environmental selection on mixed populations of microorganisms.

Intubation affects the environment of the respiratory tract, altering clearance and potentially providing a route for external microorganisms to access the lower respiratory tract. Ventilator-associated pneumonia (VAP) occurs in between $8 \%$ and $28 \%$ of intubated patients. ${ }^{7}$ The large uncertainty in this figure is at least partially explained by varying definitions of VAP in different hospitals. VAP is distinguished from hospital-acquired pneumonia (HAP) by the length of time a patient has been intubated for, though the microbiology also differs between these two acquired pneumonias. VAP is characterised by increased occurrence of infection with Gram-negative enteric and non-enteric bacilli, particularly Pseudomonas aeruginosa. $^{8}$ These organisms are also not usually found in high abundance in healthy individuals, though others responsible for acquired pneumonia, such as Streptococcus spp., Haemophilus spp. and Staphylococcus spp., can also be present in individuals without disease.

Zakharkina et $a l^{9}$ apply $16 \mathrm{~S}$ rRNA gene sequencing to a cohort of patients defined has having either VAP, HAP or community-acquired pneumonia. Patients were recruited at intubation, prior to the development of pneumonia, and VAP was defined as occurring after intubation and mechanical ventilation for $>48$ hours. They also divided patients into three further groups: those without VAP and without colonised airways; those without VAP, but who had isolates from lavage or endotracheal aspirate; and those with both VAP and an isolate. The study includes 35 subjects, a modest number given the high variance in microbial communities, but patients can be difficult to recruit in this disease group and this is greater than previous studies of the VAP microbiome. ${ }^{10}$ Crucially, they sample endotrachaeal aspirate longitudinally, collecting 127 samples. Individuality is a characteristic of the human microbiome and although common organisms occupy particular body sites, the proportions and precise membership of the microbial community from one person are unique to them. ${ }^{11} 12$ Each subject is their own best control, and longitudinal sampling through an intervention improves the power of these studies.

The authors frame their study within the adapted island model. This concept introduced to the respiratory field by Dickson, Erb-Downward and Huffnagle ${ }^{13}$ was borrowed from island ecology, where the number of different species (species richness) on an island is related to how isolated that island is from other sources of organisms, such as the mainland. The adapted island model recasts the lower respiratory tract as the island and the upper respiratory tract as the mainland. Immigration to (eg, aspiration) and emigration from (eg, cough, clearance) the island are the only factors considered by the model in defining the organisms present, which is likely to be an oversimplification. You would expect disease processes to modify the level of isolation from sources of microorganisms in the upper respiratory tract. With VAP, Zakharkina et al propose that isolation is achieved by intubation and hypothesise that this will impact diversity of organisms in the lower respiratory tract.

The authors found that diversity, measured by Shannon's index, did indeed fall with mechanical ventilation in $83 \%$ of patients, though did not alter with antibiotic treatment. Shannon's index is a way of summarising the relative proportions of each organism present in a sample and it is particularly sensitive to an abundance of rarer members of a community, so a fall suggests that an organism is becoming more dominant during mechanical ventilation. The lack of association with antibiotics is initially surprising; you might expect a substantial effect, but there were a wide range of different types used and it is unlikely that the study was powered to detect this. Both these findings are supported by independent research by other groups. ${ }^{10}$

Further analysis identified the organism increasing in dominance as Pseudomonas. This is a well-known VAP-associated organism, but what is particularly interesting and for me the crux of the paper was that this change occurred in association with mechanical ventilation, irrespective of whether the patient went on to develop VAP. To a traditional, reductionist view of microbial pathogenesis, this does 
not make much sense, virulent organisms cause disease and require treatment don't they?

It follows that exposure to and colonisation with $P$. aeruginosa is not the sole determinant in the development of VAP, but host susceptibility must also play a part. Pathogenicity and virulence have been described as emergent properties of the interaction between host and microorganism, ${ }^{14} 15$ and the results of this study would seem to support that viewpoint. The organism alone is not responsible for the pathogenic process. There are few examples of host determinants for infection, but there is evidence for this with $P$. aeruginosa itself in cystic fibrosis, where host genotype in modifier genes influences colonisation. ${ }^{16}$ In addition, the other microorganisms present, the virulence of the particular $P$. aeruginosa strains acquired and other aspects of the underlying disease are also likely to influence whether or not the colonisation becomes infection.

The implications for diagnosis and treatment of VAP are potentially profound. It would be insufficient to attempt to screen for $P$. aeruginosa or for other organisms associated with VAP, whether by culture or molecular methods. A diagnostic would need to also target undefined susceptibility determinants, in addition to the microorganisms. Conversely, additional treatment or prevention options might also present themselves with a rapid prescreen indicating the requirement for infection control measures to be put in place to attempt to limit acquisition of opportunistic pathogens by VAP-susceptible patients. They might also be flagged for non-invasive methods of mechanical ventilation, reducing isolation of the lower respiratory tract island. Antibiotic exposure and prophylaxis could be reduced in patients that were deemed resistant to VAP, with important gains to be made against antimicrobial resistance, especially given that the WHO recently identified resistant $P$. aeruginosa as a top priority for research and development. ${ }^{17}$ Further studies are required, but this research indicates that benefit for patients might be gained by considering infectious processes not simply as a result of a single infectious agent, but as the product of multiple organisms, one of which happens to be human.

\section{Twitter Follow Michael Cox @mikeyj}

Acknowledgements The author thanks Dr Leah Cuthbertson, Professor Miriam Moffatt and Professor William Cookson for discussion, to the two anonymous reviewers for their excellent suggestions and to $\mathrm{Dr}$ Tatyana Zakharkina and colleagues for a stimulating study.

Funding MJC is supported MM and WC's Wellcome Trust Joint Senior Investigator Award.

Competing interests None declared.

Provenance and peer review Commissioned; externally peer reviewed.

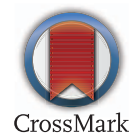

To cite Cox MJ. Thorax 2017;72:774-775.

Received 10 March 2017

Revised 4 April 2017

Accepted 6 April 2017

Published Online First 2 May 2017

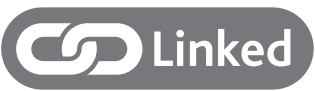

- http://dx.doi.org/10.1136/thoraxjnl-2016-209158

Thorax 2017;72:774-775

doi:10.1136/thoraxjnl-2017-209997

\section{REFERENCES}

1 Lane DJ, Pace B, Olsen GJ, et al. Rapid determination of $16 \mathrm{~S}$ ribosomal RNA sequences for phylogenetic analyses. Proc Natl Acad Sci USA 1985;82:6955-9.

2 Huang YJ, Nelson CE, Brodie EL, et al. Airway microbiota and bronchial hyperresponsiveness in patients with suboptimally controlled asthma. J Allergy Clin Immunol 2011;127:372-3.

3 Charlson ES, Chen J, Custers-Allen R, et al. Disordered microbial communities in the upper respiratory tract of cigarette smokers. PLOS ONE 2010:5:e15216

4 Cox MJ, Turek EM, Hennessy C, et al. Longitudinal assessment of sputum microbiome by sequencing of the 165 rRNA gene in non-cystic fibrosis bronchiectasis patients. PLOS ONE 2017;12:e0170622.

5 Hilty M, Burke C, Pedro H, et al. Disordered microbial communities in asthmatic airways. PLoS ONE 2010;5:e8578.

6 Erb-Downward JR, Thompson DL, Han MK, et al. Analysis of the Lung Microbiome in the "Healthy" Smoker and in COPD. PLOS ONE 2011;6:e16384.

7 Koenig SM, Truwit JD. Ventilator-associated pneumonia: diagnosis, treatment, and prevention. Clin Microbiol Rev 2006;19:637-57. doi:10.1128/ CMR.00051-05

8 Weber DJ, Rutala WA, Sickbert-Bennett EE, et al. Microbiology of ventilator-associated pneumonia compared with that of hospital-acquired pneumonia. Infect Control Hosp Epidemiol 2015:28:825-31.

9 Zakharkina T, Martin-Loeches I, Matamoros S, et al. The dynamics of the pulmonary microbiome during mechanical ventilation in the intensive care unit and the association with occurrence of pneumonia. Thorax 2017;72:803-10.

10 Kelly BJ, Imai I, Bittinger K, et al. Composition and dynamics of the respiratory tract microbiome in intubated patients. Microbiome 2016:4:7.

11 Kramer R, Sauer-Heilborn A, Welte T, et al. High individuality of respiratory bacterial communities in a large cohort of adult cystic fibrosis patients under continuous antibiotic treatment. PLOS ONE 2015;10: e0117436.

12 Zhu A, Sunagawa S, Mende DR, et al. Interindividual differences in the gene content of human gut bacterial species. Genome Biol 2015;16:82.

13 Dickson RP, Erb-Downward JR, Huffnagle GB. Towards an ecology of the lung: new conceptual models of pulmonary microbiology and pneumonia pathogenesis. Lancet Respir Med 2014;2:238-46.

14 Casadevall A, Fang FC, Pirofski LA. Microbial virulence as an emergent property: consequences and opportunities. PLoS Pathog 2011;7:e1002136.

15 Casadevall A, Pirofski LA. What is a host? Incorporating the microbiota into the damageresponse framework. Infect Immun 2015;83:2-7.

16 Park JE, Yung R, Stefanowicz D, et al. Cystic fibrosis modifier genes related to Pseudomonas aeruginosa infection. Genes Immun 2011;12:370-7.

17 World Health Organization. Global priority list of antibiotic-resistant bacteria to guide research, discovery, and development of new antibiotics. 2017:1-7. 